\title{
Implementation of an inelastic collision operator into KIPP- SOLPS coupling and its effects on electron parallel transport in the scrape-off layer plasmas
}

\author{
Menglong Zhao* ${ }^{* 1,2}$, A. V. Chankin ${ }^{1}$, and D. P. Coster ${ }^{1}$ \\ ${ }^{1}$ Max-Planck-Institut für Plasmaphysik, Garching bei München, Boltzmannstr. 2, 85748, Germany \\ ${ }^{2}$ Physik-Department E28, Technische Universität München, 85747 Garching, Germany
}

\begin{abstract}
Key words Inelastic collision operator, kinetic, fluid, edge plasmas.
This paper develops an inelastic collision operator for the KIPP code (Kinetic Code for Plasma Periphery) to investigate kinetic effects of electron cooling due to inelastic collisions. It is fully tested based on the self-consistent KIPP-SOLPS coupling algorithm by being compared to the ADAS database. The collisional radiative rate coefficients from the ADAS database for deuterium atomic physics can be recovered using the inelastic collision operator with assuming Maxwellian electrons, which shows that the inelastic collision operator works well for various plasma conditions. Across a wide range of plasma conditions in the scrape-off layer, KIPP-SOLPS coupling simulation results with the inelastic collision operator implemented are not significantly different from results that use a simpler uniform cooling scheme. The uniform scheme is thus recommended rather than including computationally intensive inelastic collision physics.
\end{abstract}

Copyright line will be provided by the publisher

\section{Introduction}

Power exhaust is one of the critical issues for future fusion devices. Currently 2D fluid codes solving Braginskiilike equations [1] are utilized to investigate tokamak edge plasmas, like SOLPS [2], EDGE2D [3], UEDGE [4]. However, the validity of the fluid model is often violated by electron parallel non-local transport $[5,6,7]$ along magnetic field lines in the scrape-off layer. Non-Maxwellian tails of electron distribution functions due to the non-local transport greatly influence electron related transport coefficients in fluid equations which are obtained by assuming collisional plasmas.

The Kinetic Code for Plasma Periphery (KIPP) [8, 9, 10, 11], which is a continuum kinetic code, solving the Vlasov-Fokker-Planck equation [12] with high accuracy of collision terms for electron parallel transport, was coupled with SOLPS based on an iterative coupling algorithm [13], as briefly described in section 2. The KIPPSOLPS coupling algorithm enables one to treat electron parallel transport fully kinetically while still keeping all the physics that SOLPS has, which would be time-consuming to capture using a kinetic code, and is accurately captured by the fluid code. In previous studies [13, 14, 15], the KIPP-SOLPS coupling algorithm was extensively investigated. The atomic physics was treated in SOLPS code and the corresponding electron cooling due to ionization, line radiation and recombination-bremsstrahlung radiation was included in KIPP by an uniform power removal scheme, as described in section 2, which was however not realistic, especially for plasmas downstream near the target. Previous kinetic simulations $[5,10,16,17,18]$ showed that the electron conductive heat flux was found to be carried mostly by the so-called Heat Carrying Electrons (HCE), which have the kinetic energy $\sim 5.95 T_{e}$. Reminding that the ionization threshold for deuterium neutrals is $13.6 \mathrm{eV}, \mathrm{HCE}$ could be mainly responsible for the deuterium ionization in low temperature region, and heat conduction coefficients therefore could be changed. In order to capture this kinetic effect, an inelastic collision operator describing electronneutral collisions is proposed in section 3 and simulations with the operator based on KIPP-SOLPS coupling algorithm are performed in section 5 .

\footnotetext{
* Corresponding author: e-mail: menglong.zhao@ipp.mpg.de,
} 


\section{KIPP-SOLPS coupling}

In order to run SOLPS with kinetic electron effects, an iterative coupling algorithm was introduced in [13]. Compared to the fluid model solved in SOLPS, this algorithm treats electron parallel transport fully kinetically by calculating four electron related kinetic factors: electron heat conduction coefficient $c_{e}$, thermal force coefficient $k_{\|}$, sheath potential drop coefficient $\delta_{\phi}$ and electron sheath heat transmission coefficient $\gamma_{e}$, with KIPP, and transferring them back to SOLPS. It can be described as 4 main steps: (1) Run SOLPS with default kinetic factors; (2) Transfer profiles of ion density and temperature, electron temperature and particle flux from SOLPS to KIPP; (3) Calculate effective kinetic factors in KIPP with maintaining the plasma profiles transferred from SOLPS; (4) transfer the effective kinetic factors back to SOLPS and run SOLPS.

\subsection{Kinetic factors in SOLPS}

A 1D version of SOLPS $[19,20]$ with forcing radial gradients to 0 , thus removing radial transport, was used for the coupling. Then the 1D spatial dimension considered in this 1D SOLPS is the poloidal direction (' $x$ '). Poloidal transport is the sum of projections of the parallel transport (' $\mid{ }^{\prime}$ ') along magnetic field lines and the transport in the diamagnetic direction (' $\perp$ ') within the magnetic flux surface on the poloidal direction. The following equations are solved for electrons in this 1D SOLPS:

$$
\begin{gathered}
\frac{\partial n_{e}}{\partial t}+\nabla_{\|}\left(\Gamma_{e \|}\right)=S_{p}-\nabla_{\perp} \Gamma_{e \perp} \\
e n_{e} E_{\|}=R_{T_{\|}}-\nabla_{\|} p_{e} \\
\frac{\partial}{\partial t}\left(\frac{3}{2} n_{e} T_{e}\right)+\nabla_{\|}\left(\frac{3}{2} n_{e} T_{e} u_{e \|}+q_{e \|}^{\text {cond }}\right) \\
=-n_{e} T_{e} \nabla_{\|} u_{e \|}-Q_{\Delta}+S_{E e}-\nabla_{\perp} q_{e \perp}
\end{gathered}
$$

where $n_{e}, u_{e \|}, T_{e}, p_{e}$ are electron density, parallel velocity, temperature, pressure, and $\Gamma_{e \|}, \Gamma_{e \perp}$ are electron parallel and perpendicular particle flux densities and $q_{e}$ is electron perpendicular heat flux density. $Q_{\Delta}$ is electron-ion energy exchange term. $E_{\|}$is the electric field along $\vec{B}$. Ambipolar transport is assumed in Eq. (2) since the analysis in this work is based on a 1D geometry (described in section 5) which enforces zero currents. The electron parallel conductive heat flux density and thermal force are given by the closure equations:

$$
\begin{aligned}
q_{e \|}^{\text {cond }} & =-c_{e} n_{e} \tau_{e} \frac{T_{e}}{m_{e}} \nabla_{\|} T_{e} \\
R_{T_{\|}} & =-k_{\|} n_{e} \nabla_{\|} T_{e}
\end{aligned}
$$

where $c_{e}=3.16, k_{\|}=0.71$ for singly charged ions.

Two boundary conditions for Eqs. (2) and (3) at the target are required in SOLPS:

$$
\begin{gathered}
\phi^{t} \quad=\delta_{\phi} \frac{T_{e}}{e} \\
q_{e \|}^{t}=\gamma_{e} T_{e} \Gamma_{t \|}
\end{gathered}
$$

where $\phi^{t}$ and $q_{e \|}^{t}$ are the potential and electron parallel heat flux density at the boundary. The potential at the target is assumed to be 0 . These two coefficients are typically $\Delta \phi \approx 3$ and $\gamma_{e} \approx 4$.

The closure equations (Eqs. (4) and (5)) and the boundary conditions (Eqs. (6) and (7)) are only valid in the collisional limit [1]. Electron non-local transport [5, 6, 7] in kinetic simulations [5, 10, 16, 17, 18, 21, 22] can significantly influence values of these four coefficients.

\subsection{Effective kinetic factors defined in KIPP}

The main equation to be solved in KIPP $[8,9,10]$ is the Vlasov-Fokker-Planck equation:

$$
\frac{\partial f_{e}}{\partial t}+v_{\|} \nabla_{\|} f_{e}-E_{\|} \frac{\partial f_{e}}{\partial v_{\|}}=\left(\frac{\partial f}{\partial t}\right)_{\text {coll. }}+S_{\mathrm{E}}+S_{\mathrm{p}}
$$


The electron distribution function $f_{e}$ is $3 \mathrm{D}$ : two dimensions in velocity space (parallel and gyro-averaged perpendicular velocity), and one dimension in physical space along the magnetic field $\vec{B} . S_{\mathrm{E}}$ and $S_{\mathrm{p}}$ are electron energy and particle sources, respectively. The collision term $\left(\frac{\partial f}{\partial t}\right)_{\text {coll. }}$ is described by the Fokker-Planck collision operator.

Four effective kinetic factors can be easily obtained in KIPP:

$$
\begin{aligned}
c_{e, e f f} & =\left(\frac{1}{2} m_{e} \int f_{e} v^{2} v_{\|} d \vec{v}-\frac{5}{2} n_{e} T_{e} u_{e \|}\right) /\left(-n_{e} \tau_{e} \frac{T_{e}}{m_{e}} \nabla_{\|} T_{e}\right) \\
k_{\|, e f f} & =\int m_{e} v^{\prime}\left(\frac{\partial f}{\partial t}\right)_{\text {coll. }} d \vec{v} /\left(-n_{e} \nabla_{\|} T_{e}\right) \\
\delta_{\phi, e f f} & =\frac{m_{e} v_{c}^{2}}{2 T_{e t}} \\
\gamma_{e, e f f} & =\frac{1}{2} m_{e} \int_{v_{c}}^{\infty} f_{t} v^{2} v_{\|} d \vec{v} /\left(T_{e t} \int_{v_{c}}^{\infty} f_{t} v_{\|} d \vec{v}\right)
\end{aligned}
$$

where $v^{\prime}=v_{\|}-\int f_{e} v_{\|} d \vec{v} / n_{e} . f_{t}, T_{e t}$ are electron distribution function, temperature at the boundary, respectively. The critical velocity $v_{c}$ is determined by the logical sheath boundary condition [23] implemented in KIPP with assuming ambipolar flows through the final boundary.

\section{Inelastic collision operator}

3.1 Default numerical schemes for particle and energy sources

The work [13] fully investigated the KIPP-SOLPS coupling algorithm, and kinetic effects of parallel electron transport were presented in [15]. However, as described in section 2, during the Step (3) of the coupling algorithm, the plasma profiles in KIPP were maintained by the automatic particle and energy source terms: $S_{\mathrm{p}}$ and $S_{\mathrm{E}}$, numerically implemented as the uniform particle and energy source schemes $F_{S}(f)$ and $F_{E}(f)$, defined in [24]:

$F_{E}(f)$ represents the process of uniform electron power input or subtraction which converts one Maxwellian into another without changing the number of particles and momentum. This is equivalent to increasing or decreasing the temperature in a fluid concept.

$F_{S}(f)$ modifies the electron density by scaling up $f_{e}$ evenly in velocity space with subsequent power removal with the uniform energy source scheme $F_{E}(f)$ to compensate for the energy content change during this process.

Numerical details of the above two terms can be found in [24].

\subsection{Inelastic collisions}

As discussed above, it is not realistic to deal with electron-neutral inelastic collisions (ionization etc.) based on the uniform source schemes in KIPP. This paper presents an inelastic collision operator for electron cooling due to deuterium ionization, line radiation, recombination and bremsstrahlung radiation.

The electron cooling due to inelastic collisions in SOLPS is calculated with the effective coefficient from ADAS database based on the collision-radiative theory [25, 26] by assuming Maxwellian electrons.

The first subsection reviews the collisional radiative theory $[25,26]$ for calculating deuterium ionization, line radiation, recombination and bremsstrahlung rate coefficients. In the second subsection, an inelastic collision operator is introduced, based on the collisional-radiative theory, for electron cooling radiation due to deuterium atomic physics, replacing the default uniform particle and energy source schemes.

\subsubsection{Collisional-radiative coefficients}

In fusion plasmas, the assumptions have been made that coronal equilibrium is held where all excited neutrals instantly transition to the ground state without experiencing any collisions with electrons by assuming that the radiative decay time from an excited state to the ground state is much shorter than the electron-neutral collision 
time. However, this assumption is often poor in the edge plasma due to the fact that substantial variations are present in the density and temperature profiles along the poloidal direction which result in much lower temperature and higher density downstream. Particularly in the divertor region where the recycling mainly occurs, the collision time is comparable to the radiative decay time, hence the metastable and excited states can exist in the ionization and recombination equilibrium, with the excited populations satisfying (see $[25,26]$ ):

$$
\frac{d n_{i}}{d t}=\sum_{j \neq i}^{\max } n_{j} C_{i j}+n_{e} n^{+} r_{i}+C_{i i} n_{i}
$$

where $i, j=1,2, \cdots, \max$, taking deuterium neutrals for example, $n_{1}$ is the density of deuterium neutrals in the ground state, $n_{i}$ is the density of deuterium neutrals in the $i^{\text {th }}$ excited state. $\max$ is the highest excited state to be considered in this work, referred to as 'cut-off level' in later discussions. $n^{+}$is the density of deuterium ions and $r_{i}$ is the direct recombination coefficient from the ion to the $i^{\text {th }}$ excited state. $C_{i j}$ is the coefficient of transition from the $j^{\text {th }}$ to $i^{\text {th }}$ states. $C_{i i}$, the loss rate due to ionization and transition from the $i^{\text {th }}$ state, is defined as:

$$
C_{i i}=-\left(\sum_{k \neq i}^{\max } C_{k i}+n_{e} S_{i}\right)
$$

$S_{i}$ is the ionization rate coefficient from the $i^{\text {th }}$ excited state.

In order to obtain an effective ionization coefficient $S^{c r}$ (collisional-radiative ionization coefficient) so that the ionization source rate is $n_{e} S^{c r} n_{n}$, where the neutral particle density $n_{n}=\sum_{k=1, \max } n_{k}$, some assumptions are made [26]:

1. The dominant populations exist in the ground state: $n_{n} \approx n_{1}$.

2. The quasi-steady state (QSS) is assumed, the neutrals in excited states are balanced by transitions, ionizations and recombinations:

$$
\frac{d \mathbf{N}_{\mathbf{j}}}{d t}=0
$$

where $\mathbf{N}_{\mathbf{j}}$ is the density array for excited states. $n_{j}$, one element in this array, is the density of deuterium neutrals in the $j^{\text {th }}$ excited state $(j=2,3, \cdots$, max $)$. Only the dominant population $n_{1}$ enters the transport equations.

From Eqs. (13) and (15), it follows:

$$
\begin{aligned}
& \mathbf{C}_{\mathbf{i} 1} n_{1}+\mathbf{C}_{\mathbf{i j}} \cdot \mathbf{N}_{\mathbf{j}}+n_{e} \mathbf{R}_{\mathbf{i}} n^{+}=0 \\
& \quad \quad \mathbf{N}_{\mathbf{j}}=-\mathbf{C}_{\mathbf{i j}}{ }^{-1} \cdot \mathbf{C}_{\mathbf{i} 1} n_{1}-n_{e} \mathbf{C}_{\mathbf{i j}}^{-1} \cdot \mathbf{R}_{\mathbf{i}} n^{+}
\end{aligned}
$$

where $\mathbf{C}_{\mathbf{i} 1}, \mathbf{C}_{\mathbf{i j}}$ are transition array and matrix; with elements $C_{\imath 1}$ and $C_{\imath j}$ designating coefficients for transition from the ground state to $i^{\text {th }}$ excited state and from $j^{\text {th }}$ to $i^{\text {th }}$ excited states, respectively; $\mathbf{R}_{\mathbf{i}}$ is the array for recombination., with one element $r_{\imath}$ designating the coefficient for direct recombination to the $i^{\text {th }}$ excited state. Combining Eq. (17) and Eq. (13) for $i=1$, instead of the ionization and recombination coefficients $S_{1}$ and $r_{1}$ in the coronal assumption, the collisional radiative rate coefficients with considering excited states as transition states can be obtained [25]:

$$
\begin{aligned}
S^{c r} & =S_{1}-\mathbf{S}_{\mathbf{j}} \cdot\left(\mathbf{C}_{\mathbf{i j}}{ }^{-1} \cdot \mathbf{C}_{\mathbf{i} \mathbf{1}}\right) \\
R^{c r} & =r_{1}-\mathbf{C}_{\mathbf{1} \mathbf{j}} \cdot\left(\mathbf{C}_{\mathbf{i j}}{ }^{-1} \cdot \mathbf{R}_{\mathbf{i}}\right) \\
Q^{c r} & =\left(C_{11}-\mathbf{C}_{\mathbf{1 j}} \cdot\left(\mathbf{C}_{\mathbf{i j}}{ }^{-1} \cdot \mathbf{C}_{\mathbf{i} \mathbf{1}}\right)\right) / n_{e}
\end{aligned}
$$

where $S^{c r}$ is the collisional radiative ionization rate coefficient, $R^{c r}$ is the collisional radiative recombination rate coefficient and $Q^{c r}$ is the cross-coupling coefficient. Moreover, in deuterium plasmas, the quasi-neutrality and quasi-steady state result in (see [26]):

$$
\frac{d n_{e}}{d t}=\frac{d n^{+}}{d t}=-\frac{d n_{1}}{d t}
$$


$S^{c r}$ and $Q^{c r}$ are the effective ionization rate coefficient from the ground state and the net loss coefficient from the ground state. Therefore,

$$
S^{c r}=-Q^{c r}
$$

should be fulfilled. This equation can be used to test the implementation of the collisional radiative model in the coupling algorithm.

The electron cooling due to inelastic collisions, regarded as electron energy sink due to inelastic collisions of electrons with neutrals in the energy equation in SOLPS, can then be derived [25]:

$$
P=P^{\text {io }}+P^{\mathrm{lt}}+P^{\mathrm{br}}+P^{\mathrm{rec}}
$$

where

$$
\begin{aligned}
P^{\mathrm{io}} & =I_{\mathrm{th}} S^{c r} n_{e} n_{1} \\
P^{\mathrm{lt}} & =\sum_{k=1, \text { max }-1}^{j>k} \Delta E_{j \rightarrow k} A_{k j} n_{j}^{1} \\
P^{\mathrm{rec}} & =-I_{\mathrm{th}} R^{\text {cr, coll }} n_{e} n_{1} \\
P^{\mathrm{br}} & =\sum_{k=1, \text { max }-1}^{j>k} \Delta E_{j \rightarrow k} A_{k j} n_{j}^{+}
\end{aligned}
$$

are ionization, line, recombination and Bremsstrahlung radiation power rates respectively. $I_{\mathrm{th}}=13.6 \mathrm{eV}$. $\Delta E_{j \rightarrow k}$ is the energy difference between levels $j$ and $k . n_{j}^{1}$ and $n_{j}^{+}$are elements of the two arrays: $\mathbf{N}_{\mathbf{j}}^{1}$ and $\mathbf{N}_{\mathbf{j}}^{+}$, defined respectively $(j=2,3, \cdots, \max )$ as:

$$
\begin{gathered}
\mathbf{N}_{\mathbf{j}}^{\mathbf{1}}=-\mathbf{C}_{\mathbf{i j}}{ }^{-1} \cdot \mathbf{C}_{\mathbf{i} 1} n_{1}=\boldsymbol{\alpha}_{\mathbf{j}} n_{1} \\
\mathbf{N}_{\mathbf{j}}^{+}=-n_{e} \mathbf{C}_{\mathbf{i j}}{ }^{-1} \cdot \mathbf{R}_{\mathbf{i}} n^{+}=\boldsymbol{\beta}_{\mathbf{j}} n^{+}
\end{gathered}
$$

where $\mathbf{N}_{\mathbf{j}}^{1}$ and $\mathbf{N}_{\mathbf{j}}^{+}$are two components of $\mathbf{N}_{\mathbf{j}}$. $\alpha_{j}$, one element in array $\boldsymbol{\alpha}_{\mathbf{j}}$, and $\beta_{j}$, one element in array $\boldsymbol{\beta}_{\mathbf{j}}$, are weight factors.

\subsubsection{Inelastic collision operator for e-n collisions}

The collisional-radiative processes are shown in Fig. 1 (two excited levels as an example). The neutrals at $j^{\text {th }}$ excited state have two components: $n_{j}^{1}$ due to excitations from $n_{1}$ and $n_{j}^{+}$due to recombinations from $n^{+}$. Only deuterium neutral ionization and line radiation power sinks, which are the atomic processes related to $\mathbf{N}_{\mathbf{j}}^{1}$ (inside of the red dashed box of Fig. 1), are considered in this inelastic collision operator since the recombination and Bremsstrahlung radiation power rates are comparatively negligible in the electron temperature region $1 \sim 100 \mathrm{eV}$. The rate coefficients for atomic processes related to $\mathbf{N}_{\mathbf{j}}^{+}$(inside of the green dashed box of Fig. 1) are directly taken from the ADAS database for saving CPU time.

The electron cooling radiation due to ionization is $I_{\mathrm{th}} S^{c r} n_{e} n_{1}$, hence there should be equal number of neutrals $S^{c r} n_{e} n_{1}$ in the ground state being ionized. However, it doesn't mean that there would be the same number of electrons with each losing energy of the amount of $I_{\mathrm{th}}$, since one neutral might be ionized by multiple collisions with different electrons. Therefore, Eqs. (24) and (25) are not used to derive the inelastic collision operator. They can be transformed into the sum of the energy loss due to direct excitation, de-excitation or radiative transition and ionization processes:

$$
P^{\mathrm{io}}+P^{\mathrm{lt}}=\sum_{j} \frac{I_{\mathrm{th}}}{j^{2}} S_{j} n_{e} \alpha_{j} n_{1}+\sum_{j, i}^{j \neq i} \frac{I_{\mathrm{th}}}{j^{2}-i^{2}} k_{i j} n_{e} \alpha_{j} n_{1}
$$

where $k_{i j}$ has the similar meaning of $C_{i j}$ :

$$
C_{i j}=k_{i j} n_{e}
$$




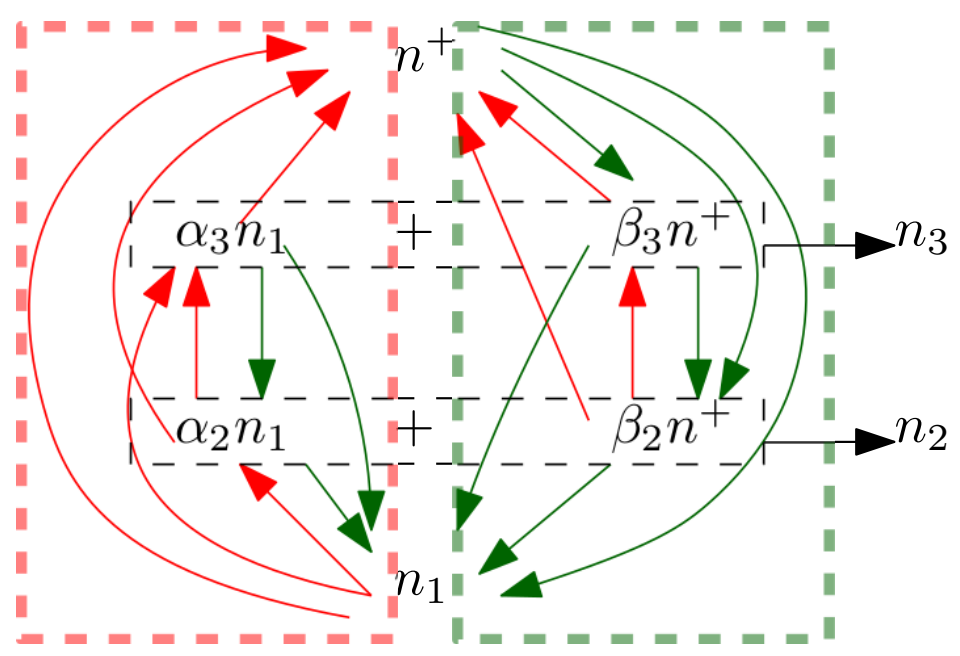

Fig. $1 n_{1}$ is the deuterium neutral density in the ground state. $n^{+}$is the deuterium ion density. The neutral densities in the second and third excited states are: $n_{2}=\alpha_{2} n_{1}+\beta_{2} n^{+}$and $n_{3}=\alpha_{3} n_{1}+\beta_{3} n^{+}$(from Eqs. (28) and (29)). The red arrows are the ionization and excitation processes while the green arrows are the recombination and deexcitation processes. The atomic processes in the red (green) dashed boxes are related to ionization and line radiation (recombination and Bremsstrahlung radiation).

Each of the direct processes can be easily described by an inelastic collision operator. It is written as:

$$
\left(\frac{\partial f}{\partial t}\right)_{\text {inc }}=\sum_{j}\left(\frac{\partial f}{\partial t}\right)_{j}^{\text {io }}+\sum_{i, j}\left(\frac{\partial f}{\partial t}\right)_{i, j}^{\mathrm{ex}, \mathrm{de}}
$$

where the inelastic collision operator $\left(\frac{\partial f}{\partial t}\right)_{j}^{\text {io }}$ due to ionization of deuterium neutrals at level $j$ and $\left(\frac{\partial f}{\partial t}\right)_{i, j}^{\text {ex,de }}$ due to the excitation or de-excitation from levels $j$ to $i$ are defined respectively as:

$$
\begin{gathered}
\left(\frac{\partial f(\vec{v})}{\partial t}\right)_{j}^{\text {io }}=-\sigma^{j \rightarrow+}|\vec{v}| f(\vec{v}) \alpha_{j} n_{1} \\
\left(\frac{\partial f\left(\overrightarrow{v^{\prime}}\right)}{\partial t}\right)_{j}^{\text {io }}=\sigma^{j \rightarrow+}|\vec{v}| f(\vec{v}) \alpha_{j} n_{1} d \vec{v} / d \overrightarrow{v^{\prime}} \\
\left(\frac{\partial f(\vec{v})}{\partial t}\right)_{i, j}^{\text {ex,de }}=-\sigma^{j \rightarrow i}|\vec{v}| f(\vec{v}) \alpha_{j} n_{1} \\
\left(\frac{\partial f\left(\overrightarrow{v^{\prime}}\right)}{\partial t}\right)_{i, j}^{\text {ex,de }}=\sigma^{j \rightarrow i}|\vec{v}| f(\vec{v}) \alpha_{j} n_{1} d \vec{v} / d \overrightarrow{v^{\prime}}
\end{gathered}
$$

where $\vec{v}$ is the velocity of the electrons before inelastic collisions with deuterium neutrals and $\overrightarrow{v^{\prime}}$ is the velocity of the scattered electrons. For the operator of ionization from level $j,{\overrightarrow{v^{\prime}}}^{2}=\vec{v}^{2}-2 I_{\mathrm{th}} / j^{2}$ and for that of excitation or de-excitation from levels $j$ to $i,{\overrightarrow{v^{\prime}}}^{2}=\vec{v}^{2}-2 I_{\mathrm{th}} /\left(j^{2}-i^{2}\right)$. Due to the lack of the differential cross section data of the scattered electron velocity angle, it is better to assume the scattering angle as evenly distributed, however such an assumption requires extremely large computational time. In this work, it is assumed that $\overrightarrow{v^{\prime}} \| \vec{v}$. The 
inelastic collision operator should automatically satisfy:

$$
\begin{array}{r}
\frac{1}{2} m_{e} \int \vec{v}^{2}\left(\frac{\partial f}{\partial t}\right)_{\mathrm{inc}} d \vec{v}=-I_{\mathrm{th}} S^{c r} n_{e} n_{1}-\sum_{k, j}^{j>k} \Delta E_{j \rightarrow k} A_{k j} \alpha_{j} n_{1} \\
\Longrightarrow-\frac{1}{n_{e} n_{1}} \frac{m_{e}}{2} \int \vec{v}^{2}\left(\frac{\partial f}{\partial t}\right)_{\mathrm{inc}} d \vec{v}=\left(I_{\mathrm{th}} S^{c r}+\frac{1}{n_{e}} \sum_{k, j}^{j>k} \Delta E_{j \rightarrow k} A_{k j} \alpha_{j}\right)
\end{array}
$$

which can be used to test the numerical implementation. $\sum_{k, j}^{j>k} \Delta E_{j \rightarrow k} A_{k j} \alpha_{j}$ is the line radiation coefficient. The electron cooling due to recombination and Bremsstrahlung radiation is included using the uniform energy source scheme $F_{E}(f)$.

\section{Numerical validation of the inelastic operator}

Non-Maxwellian effects of electron distribution functions on the effective ionization rate coefficient $S^{c r}$ in selfconsistent KIPP-SOLPS coupling simulations were investigated in [15], where the cut-off level $\max =10$ was found to give good agreement between the rate coefficient $S_{c r}$ calculated from Eq. (18) with Maxwellian electron distribution function $f_{M}$ and that taken from the ADAS database, as shown in Fig. 2. The comparison between line radiation coefficient $\sum_{k, j}^{j>k} \Delta E_{j \rightarrow k} A_{k j} \alpha_{j}$ calculated with $\max =10$ and $f_{M}$ and that from the ADAS database is also shown in Fig. 2. The good agreement indicates that the cut-off level max $=10$ is good enough to be used for implementing the inelastic collision operator.

Validation of successful implementation of the inelastic collision operator requires that Eq. (37) should be satisfied. Fig. 3 shows the comparison between the power sinks using the inelastic operator (the term on the left hand side of Eq. (37)) and that from ADAS database (the term on the right hand side of Eq. (37)) for various plasma densities $\left(10^{18} \sim 10^{21} \mathrm{~m}^{-3}\right)$ and temperatures $(1 \sim 100 \mathrm{eV})$. The power loss due to the direct ionization from the ground state using the inelastic collision operator, as a comparison, is shown as well. It can be clearly seen that the inelastic collision operator works well for various plasma conditions covering the edge.

\section{Simulations}

In order to investigate kinetic effects of electron cooling due to inelastic collisions, KIPP-SOLPS coupling runs with the inelastic collision operator are performed.

\subsection{Setup}

The coupling simulation geometry is taken from [13], which is a 1D rectangular box with the "stagnation point" and the "target" as left and right boundaries, corresponding to a flux tube from the outer mid-plane to the target with poloidal length $L_{p o l}=2.5 \mathrm{~m}$ and parallel length $L_{p a r} \approx 25 \mathrm{~m}$. There are 127 cells non-uniformly distributed, with coarser cells upstream and finer cells near the target.

The deuterium ion density at the stagnation point is scanned from $n_{u}=0.5 \times 10^{19} \mathrm{~m}^{-3}$ to $n_{u}=2.5 \times 10^{19} \mathrm{~m}^{-3}$ with the aim at varying the upstream collisionalities from low to high. The power input is evenly distributed upstream from the stagnation point to $\sim \frac{1}{3}$ of the simulation domain. It is equally split between the electron and ion channels.

As in [15], atomic rate coefficients related to ionization and line radiation are calculated by Eqs. (24) and (25). But in this work, the corresponding electron cooling radiation removal in KIPP are described by two numerical schemes:

Approach A: removing the radiation from the velocity cells using the uniform energy source scheme $F_{E}(f)$ (as described in 3.1).

Approach B: removing the radiation using the inelastic collision operator (Eq. (32)) with $f_{e}$ calculated in KIPP (as described in 3.2). 


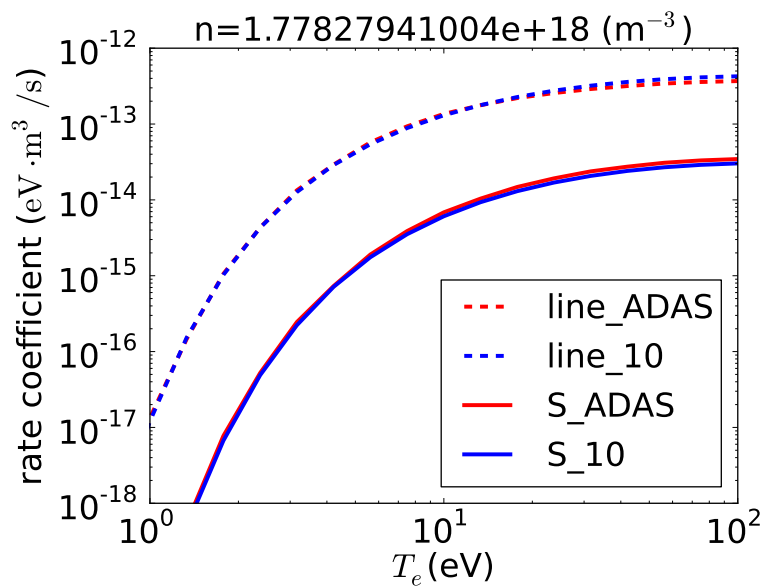

(a)

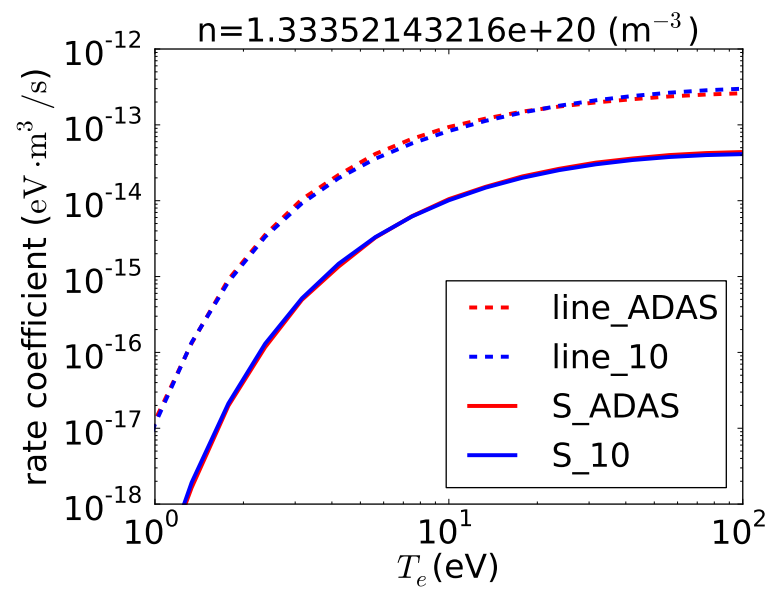

(c)

Fig. 2 The deuterium neutral ionization ('S') and line radiation ('line') rate coefficients calculated from Eq. (30) with $\max =$ 10 and Maxwellian electron distributions (designated as '10') are compared to the ADAS database (designated as 'ADAS') for various electron densities: (a) $\sim 10^{18}$, (b) $\sim 10^{19}$, (c) $\sim 10^{20}$, (d) $\sim 10^{21} \mathrm{~m}^{-3}$. The cut-off level $\max =10$ gives almost the same results as the ADAS database in the electron temperature range $\left(T_{e}<\sim 100 \mathrm{eV}\right)$ that already covers the edge electron temperature.

$\max =10$ is used as the cut-off level since it is good enough, as discussed before, for the consideration of multiple inelastic collisions while keeping the inelastic collision operator reasonably efficient.

The schematic flowchart of the KIPP-SOLPS coupling runs is shown in Fig. 4, similar to that described in section 2, but replacing the ADAS data for deuterium ionization and line radiation coefficients with Eqs. (24) and (25) with the cut-off level $\max =10$ in SOLPS, and removing the corresponding electron cooling radiation due to inelastic collisions with the uniform scheme (Approach A) or the inelastic collision operator (Approach B) in KIPP.

\subsection{Results}

Figs. 5 and 6 show that the steady state profiles from the case with the uniform energy scheme (Approach A) are exactly the same as those with the inelastic collision operator (Approach B) for the medium collisionality case with $n_{u}=1.5 \times 10^{19} \mathrm{~m}^{-3}$. This conclusion can be applied to all scanned cases with various collisionalities we run. The reason for this is similar to the argument in [24] that the electron cooling sink is comparatively more 


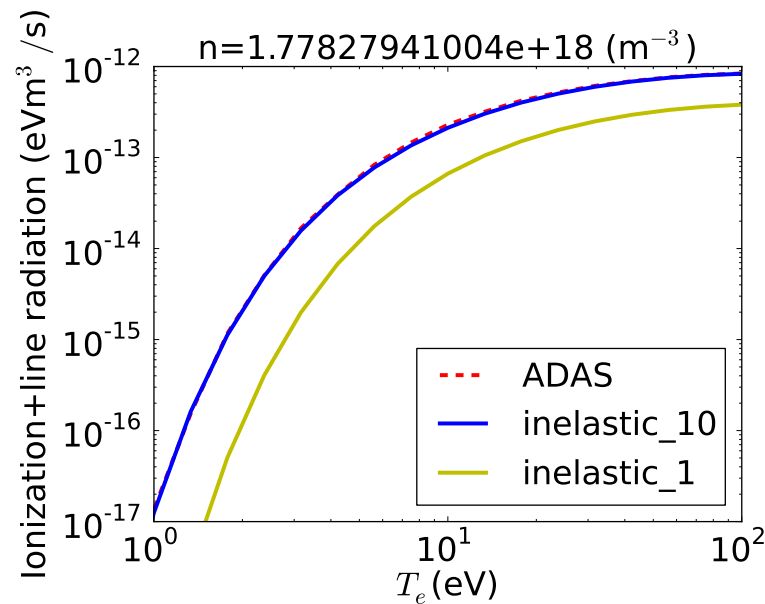

(a)

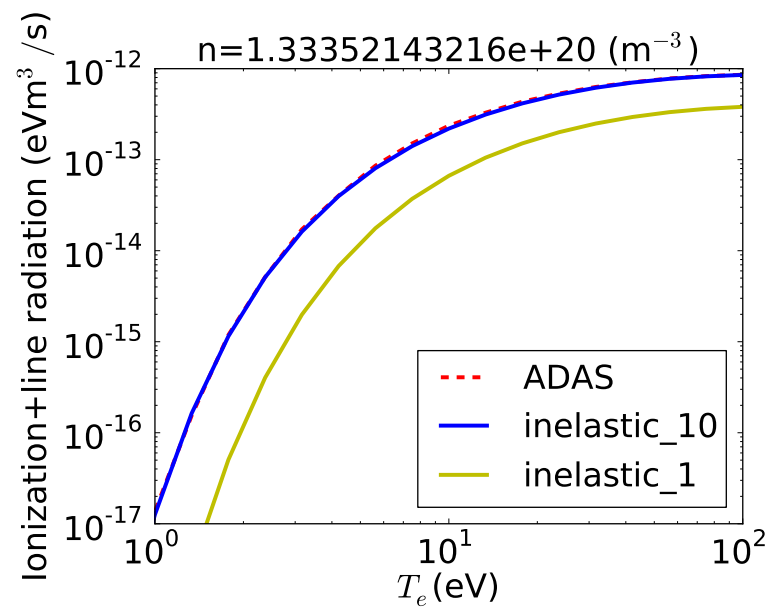

(c)

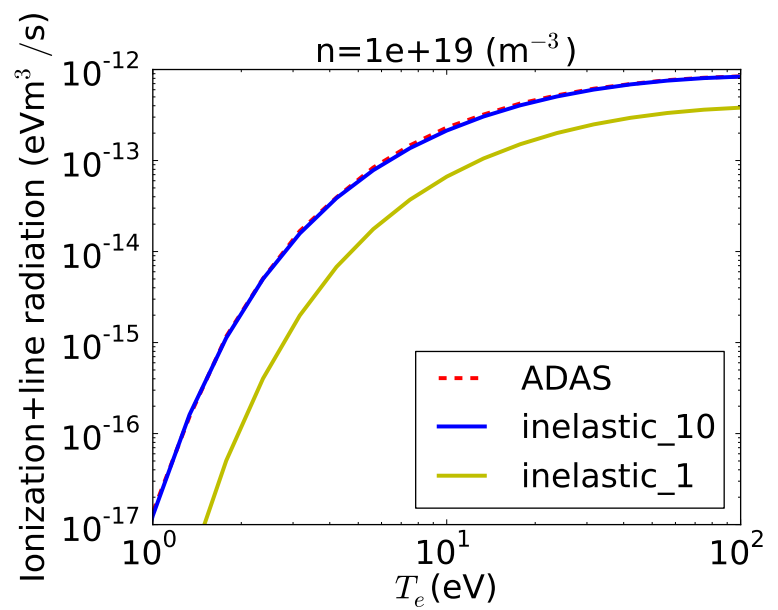

(b)

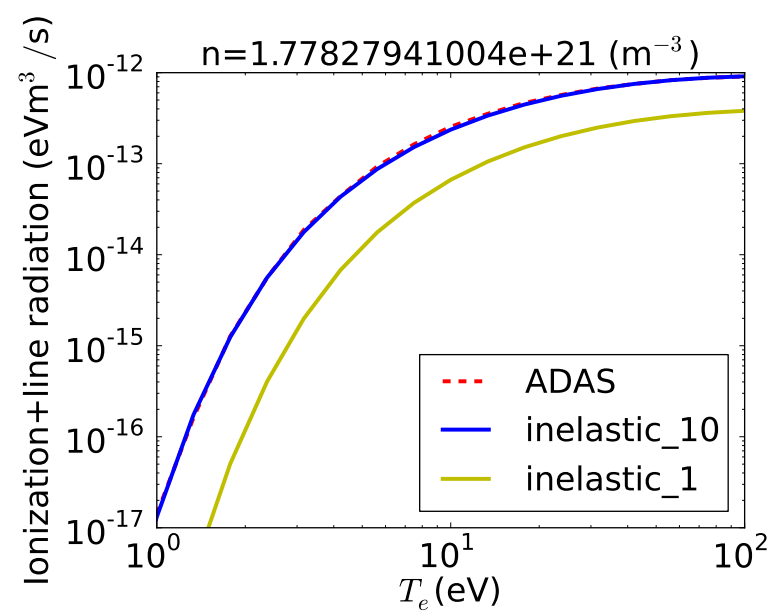

(d)

Fig. 3 Comparison between electron cooling rate due to ionization and line radiation calculated using the inelastic collision operator with $\max =10$ ('inelastic_10') and that from the ADAS database ('ADAS') for various electron densities: (a) $\sim 10^{18}$, (b) $\sim 10^{19}$, (c) $\sim 10^{20}$, (d) $\sim 10^{21} \mathrm{~m}^{-3}$. 'inelastic_1' designates the electron cooling rate calculated based on the inelastic collision operator with only considering the ground state, as a comparison.

symmetric in the velocity space, although the model used there was not self-consistent and the radiation sink was artificial, which, however, captured the main physical picture.

With respect to this medium collisionality, in the steady state, the power balance at cell $111(x \approx 2.494 \mathrm{~m})$, where the radiation is mostly concentrated, among all contributed terms is shown in Fig. 7. It can be clearly seen that the two dominant terms are the free streaming and radiation terms:

$$
\int \Delta f_{e}^{\mathrm{fs}} \vec{v}^{2} d \vec{v}+\int \Delta f_{e}^{\mathrm{rad}} \vec{v}^{2} d \vec{v} \approx 0
$$

where $\Delta f_{e}^{\mathrm{fs}}$ and $\Delta f_{e}^{\mathrm{rad}}$ are the the distribution function changes at cell 111 due to the free streaming and radiation terms, respectively, while advancing $\Delta t$ (one time step) in time. One would expect to see changes of the balance of HCE introduced by changing the numerical scheme of radiation from the uniform one (Approach A) to the inelastic collision operator (Approach B), which was expected to modify the heat conduction coefficient and thereafter the electron temperature profile. This however is not the case. The distributions of $\Delta f_{e}^{\mathrm{fs}}$ and $\Delta f_{e}^{\mathrm{rad}}$ in the velocity space are shown in Fig. 8. $\Delta f_{e}^{\mathrm{fs}}$ is rather asymmetric compared with $\Delta f_{e}^{\mathrm{rad}}$ by comparing Figs. 8a 


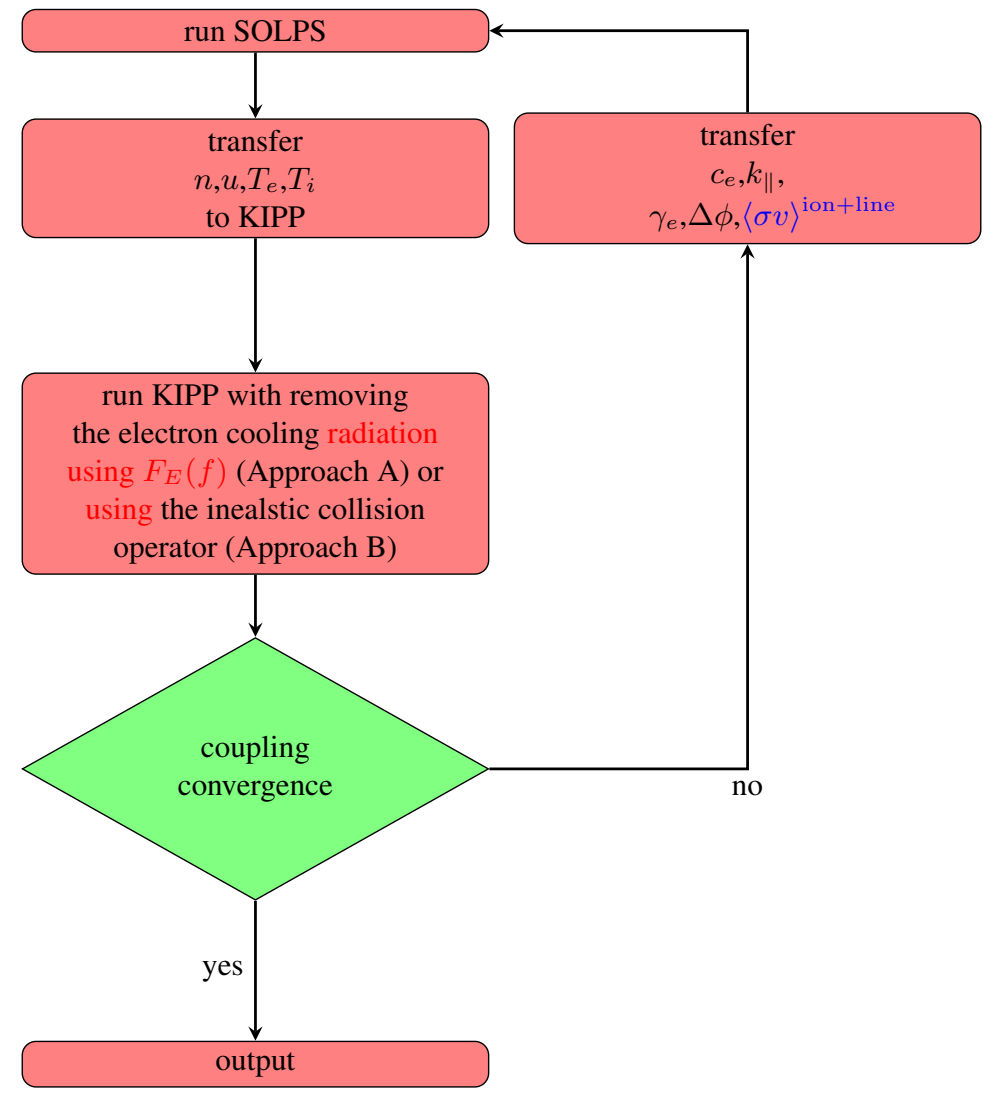

Fig. 4 The coupling algorithm with kinetic effects on D neutral ionization and line radiation.

and $8 \mathrm{~b}$ or Figs. $8 \mathrm{c}$ and $8 \mathrm{~d}$. Obviously $\Delta f_{e}$ due to the free streaming term in the cells around $\operatorname{HCE}\left(v_{\|}=2.82 v_{\mathrm{th}}\right.$, $v_{\perp}=1.98 v_{\mathrm{th}}$, where $v_{\mathrm{th}}=\sqrt{T_{e} / m_{e}}$, see [10]) is not balanced by the radiation term (inelastic collisions) but by the Coulomb collision term:

$$
\Delta f_{e}^{\mathrm{fs}}(\mathrm{HCE})+\Delta f_{e}^{\mathrm{c}}(\mathrm{HCE}) \approx 0
$$

with

$$
\left|\Delta f_{e}^{\mathrm{rad}}(\mathrm{HCE})\right|<<\left|\Delta f_{e}^{\mathrm{fs}}(\mathrm{HCE})\right|
$$

This indicates that the change of the numerical scheme for the electron cooling radiation has negligible effects on the distribution of HCE for all collisionalities. To understand Eq. (40), it is helpful to introduce inelastic and Coulomb collision times for HCE, as defined in the following equations:

$$
\begin{aligned}
\tau_{\mathrm{HCE}}^{\mathrm{inc}} & =\frac{1}{\sum_{j} \sigma^{j \rightarrow+} v_{\mathrm{HCE}} \alpha_{j} n_{1}+\sum_{i, j} \sigma^{j \rightarrow i} v_{\mathrm{HCE}} \alpha_{j} n_{1}} \\
\tau_{\mathrm{HCE}}^{\mathrm{c}} & =3(2 \pi)^{3 / 2} \frac{\varepsilon_{0}^{2} m_{e}^{1 / 2} T_{\mathrm{HCE}}^{3 / 2}}{n_{i} Z_{i}^{2} e^{4} \ln \Lambda}
\end{aligned}
$$

where $v_{\mathrm{HCE}}$ and $T_{\mathrm{HCE}}$ are $\mathrm{HCE}$ velocity and temperature. $v_{\mathrm{HCE}}=\sqrt{2.82^{2}+1.98^{2}} v_{\mathrm{th}}$. Comparison between HCE collision times due to Coulomb collisions $\left(\tau_{\mathrm{HCE}}^{\mathrm{c}}\right)$ and inelastic collisions $\left(\tau_{\mathrm{HCE}}^{\mathrm{inc}}\right)$ are shown in Fig. 9 for varying electron densities and temperatures. Since the HCE inelastic collision time is the function of the neutral density $n_{1}$, a wide variation of different neutral densities: $2 \times 10^{18} \mathrm{~m}^{-3}, 2 \times 10^{19} \mathrm{~m}^{-3}, 2 \times 10^{20} \mathrm{~m}^{-3}, 2 \times 10^{21} \mathrm{~m}^{-3}$ 

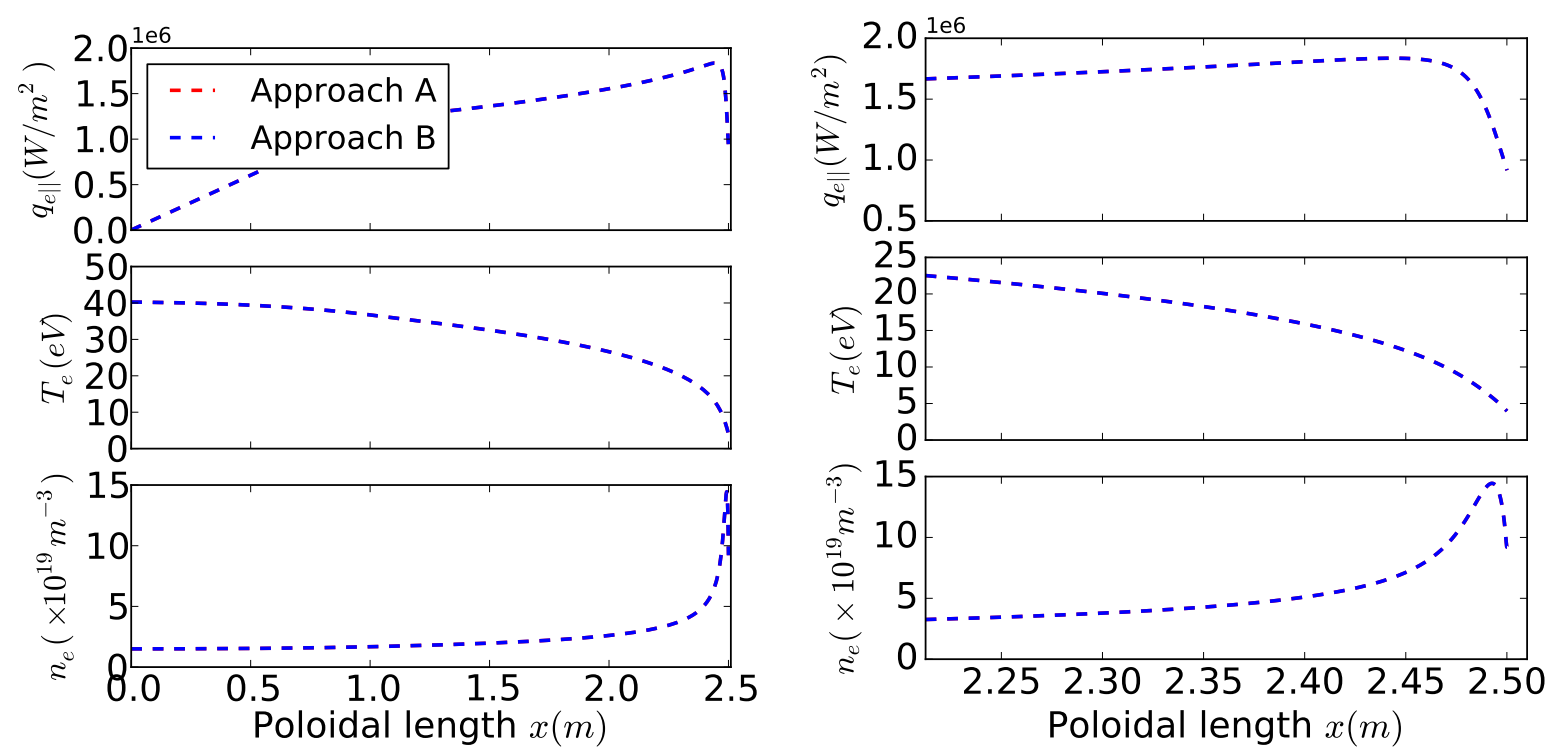

(a) $n_{u}=1.5 \times 10^{19} \mathrm{~m}^{-3}$

(b) Blow-up of the region $x=2.2 \sim 2.5 \mathrm{~m}$.

Fig. 5 Comparison between the steady state profiles of electron density $n_{e}$, temperature $T_{e}$ and heat flux density $q_{e} \|$ in Approaches A and B for the medium collisionality case with $n_{u}=1.5 \times 10^{19} \mathrm{~m}^{-3}$. (b) is the blow-up of the region near the target in (a).
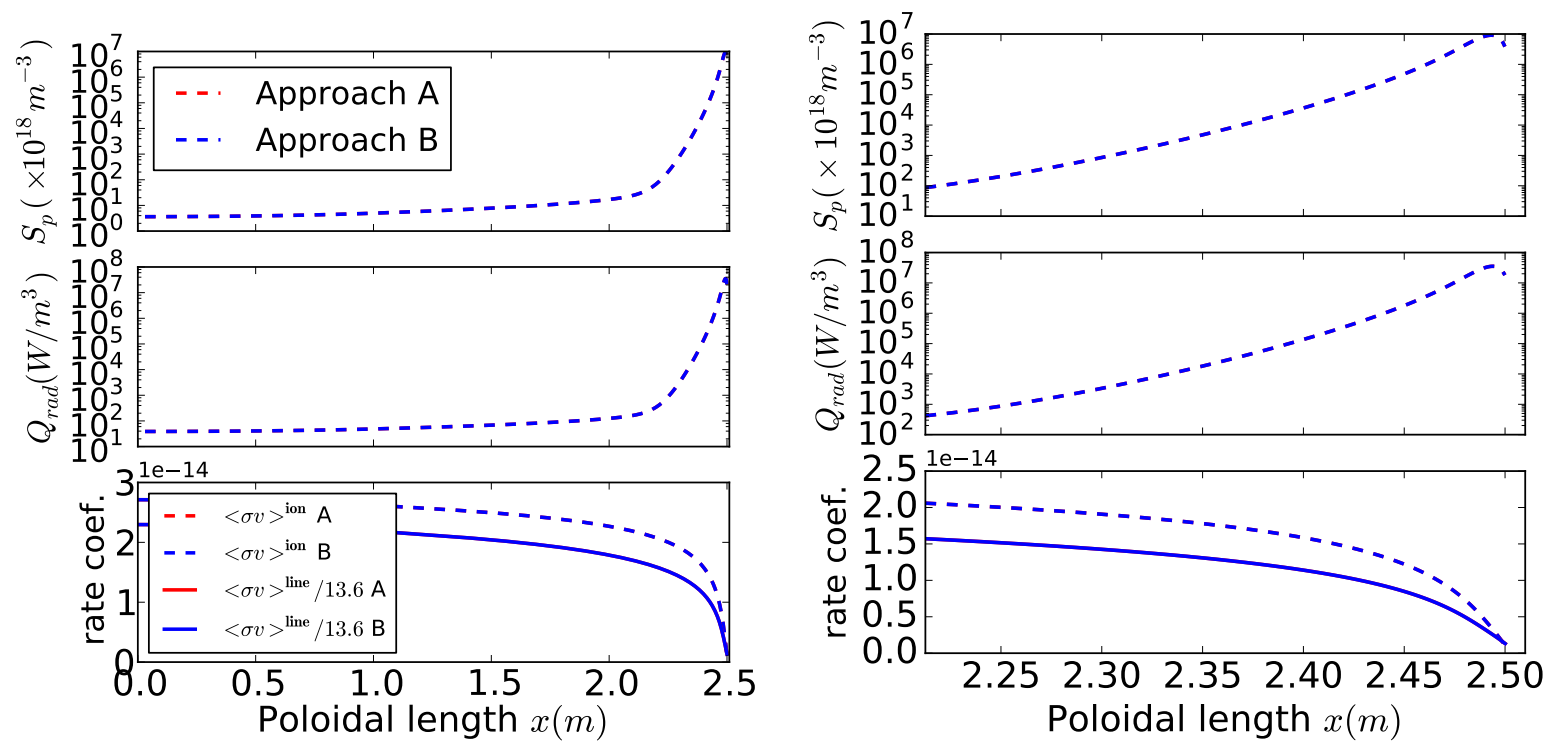

(a) $n_{u}=1.5 \times 10^{19} \mathrm{~m}^{-3}$

(b) Blow-up of the region $x=2.2 \sim 2.5 \mathrm{~m}$.

Fig. 6 Comparison between the steady state profiles of ionization particle sources $S_{p}$, radiation energy sinks $Q_{r a d}$ and ionization, line radiation rate coefficients in Approaches $\mathrm{A}$ and $\mathrm{B}$ for the medium collisionality case with $n_{u}=1.5 \times$ $10^{19} \mathrm{~m}^{-3}$. (b) is the blow-up of the region near the target in (a). 


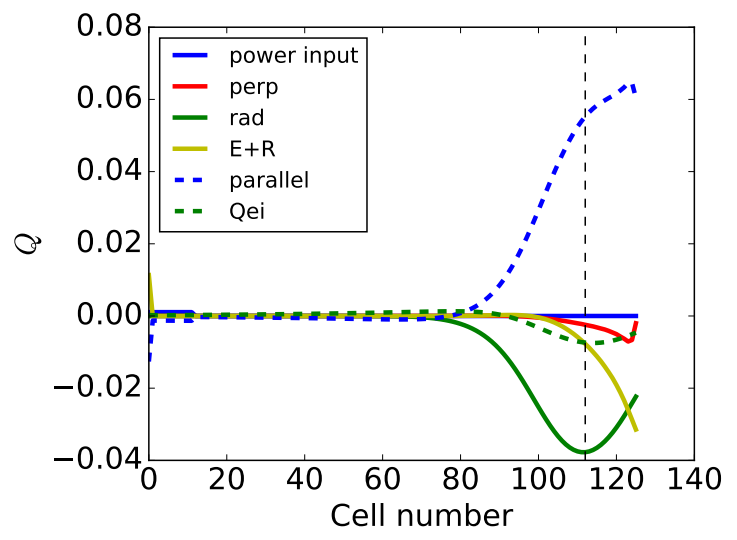

(a) All cells.

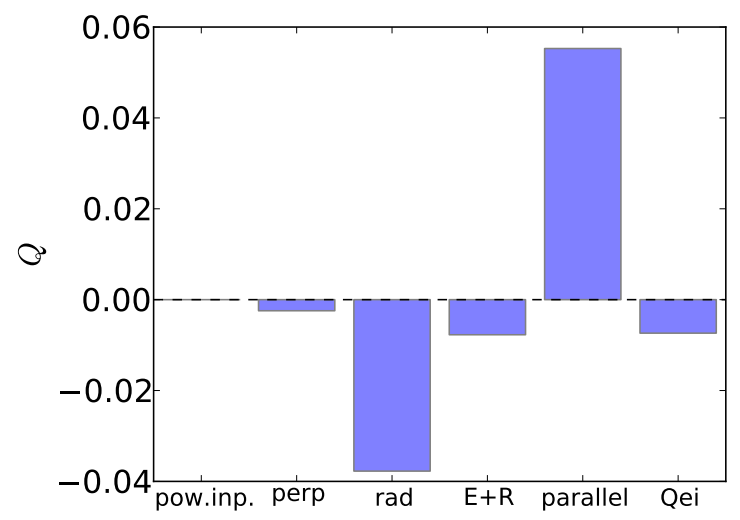

(b) Cell $111(x \approx 2.494 \mathrm{~m})$.

Fig. 7 Energy balance between the six terms: power input (blue solid), perpendicular heat flux (red solid), radiation (green solid), electric field and thermal force (yellow solid), parallel heat flux (blue dashed), heat exchange (green dashed), are shown for all cells (a) and cell 111 (b). The free-streaming term is mainly balanced by the radiation term at cell 111.

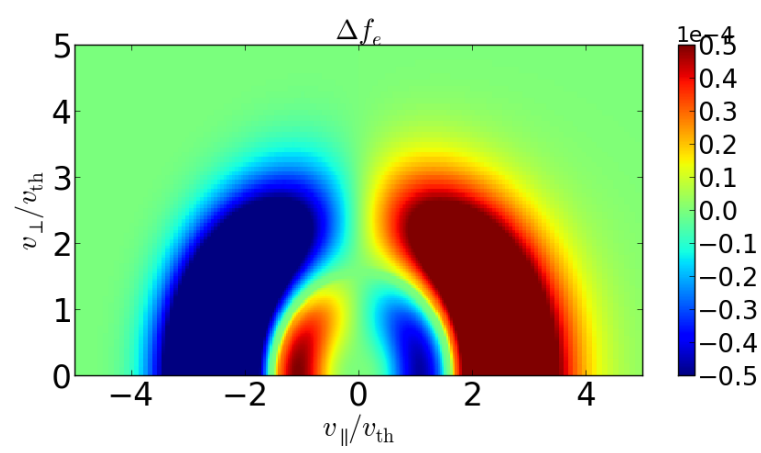

(a) $\Delta f_{e}$ due to the free-streaming term in Approach A.

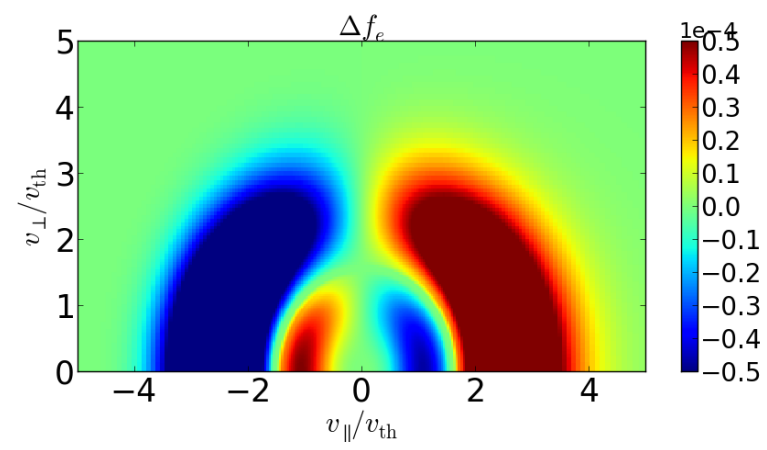

(c) $\Delta f_{e}$ due to the free-streaming term in Approach B.

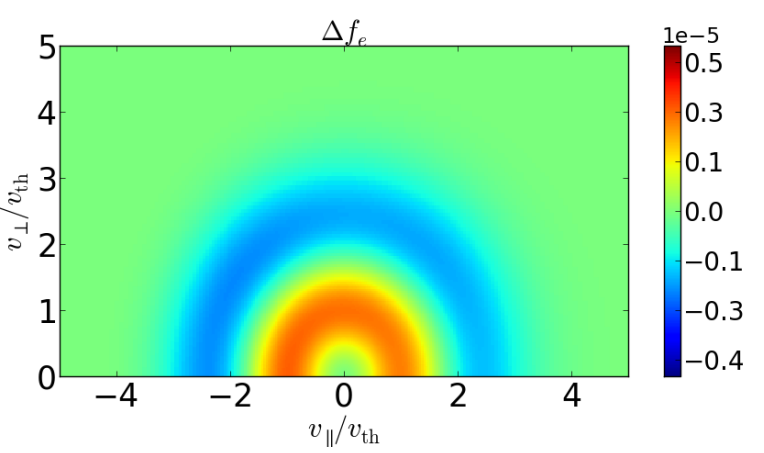

(b) $\Delta f_{e}$ due to the radiation sink in Approach A.

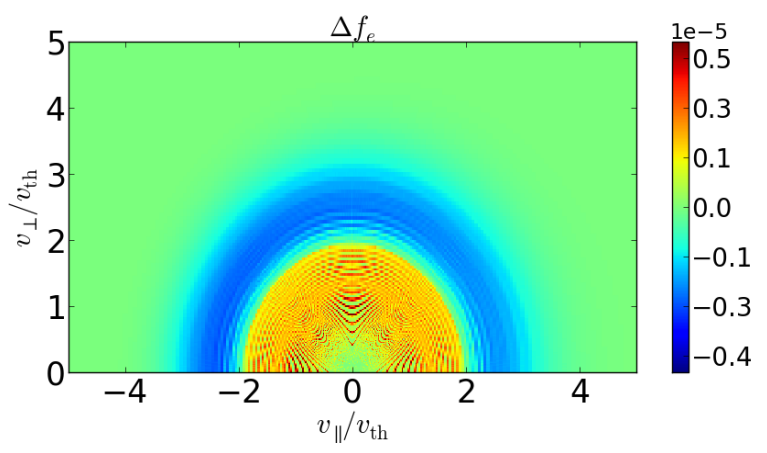

(d) $\Delta f_{e}$ due to the radiation sink in Approach B.

Fig. 8 The change of the distribution function $\left(\Delta f_{e}\right)$ due to the free-streaming $(\mathrm{a}, \mathrm{c})$ and radiation $(\mathrm{b}, \mathrm{d})$ terms with advancing $\Delta t$ (one time step) at cell 111.

is used to calculate $\tau_{\mathrm{HCE}}^{\mathrm{inc}}$ for every pair of $n_{e}$ and $T_{e}$. At cell 111 where $n_{e} \approx 1.4 \times 10^{20} \mathrm{~m}^{-3}, T_{e} \approx 5.5 \mathrm{eV}$ and $n_{1} \approx 2.0 \times 10^{19} \mathrm{~m}^{-3}$ (denoted by the vertical dashed line in Fig. 9):

$$
\tau_{\mathrm{HCE}}^{\mathrm{c}} \ll \tau_{\mathrm{HCE}}^{\mathrm{inc}}
$$




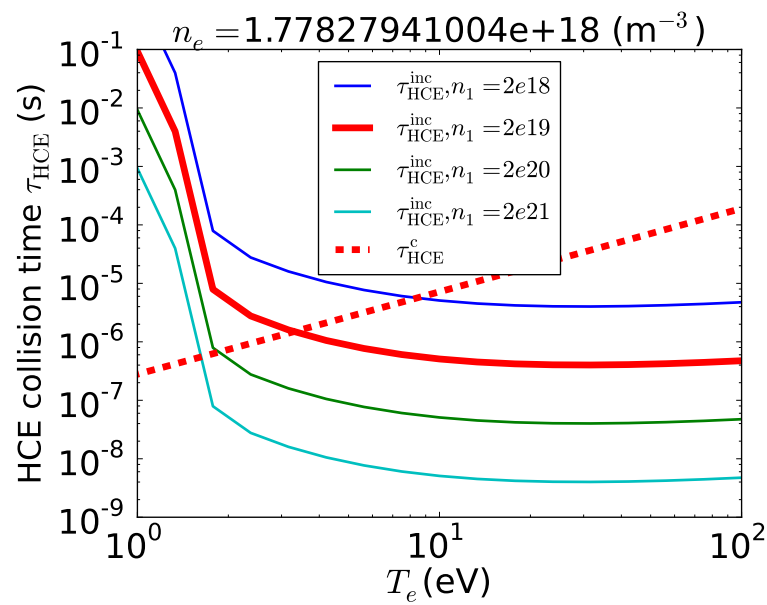

(a)

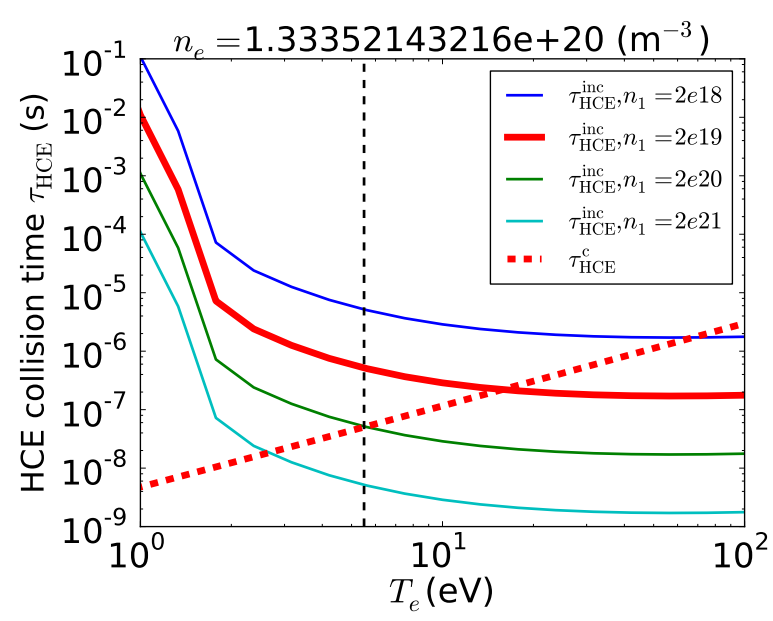

(c)

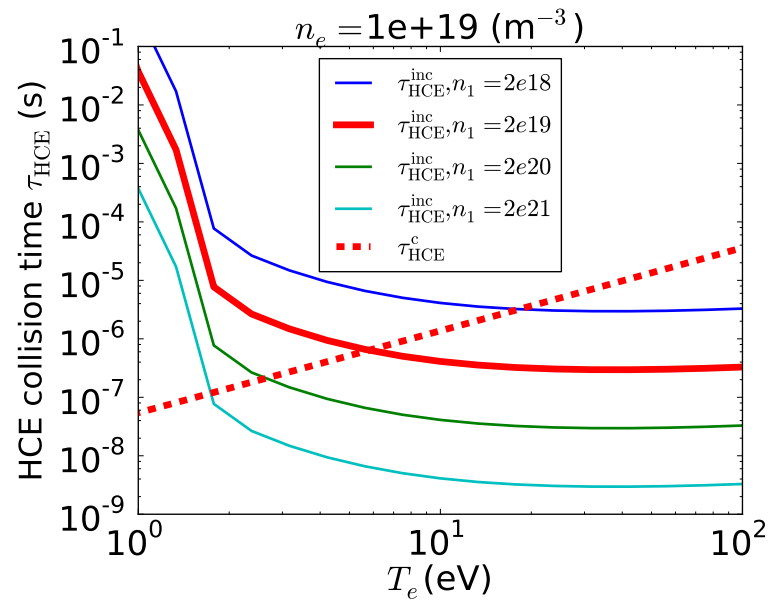

(b)

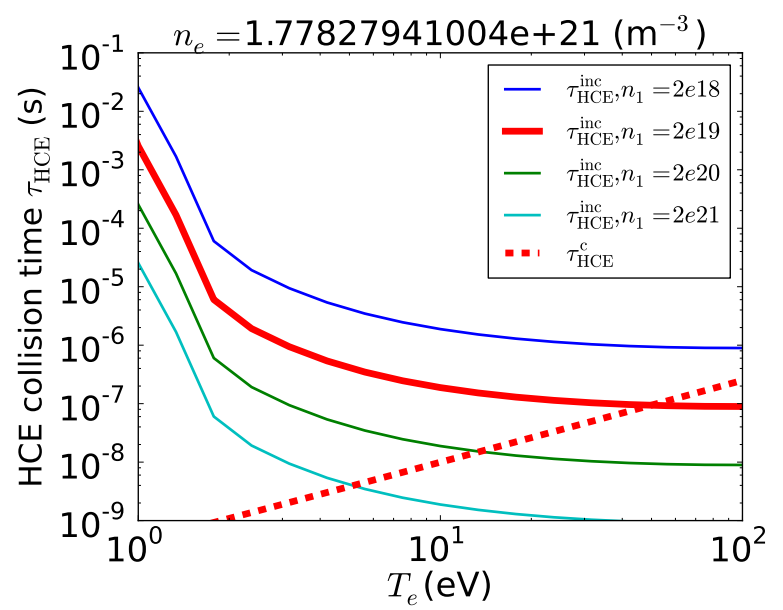

(d)

Fig. 9 Comparison between collision times for HCE due to inelastic collisions ( $\left.\tau_{\mathrm{HCE}}^{\text {inc }}\right)$ and Coulomb collisions $\left(\tau_{\mathrm{HCE}}^{\mathrm{c}}\right)$, as defined in Eqs. (41) and (42), against electron temperature $T_{e}$ for various electron densities: (a) $\sim 10^{18}$, (b) $\sim 10^{19}$, (c) $\sim$ $10^{20}$, (d) $\sim 10^{21} \mathrm{~m}^{-3}$. Four neutral densities: $2 \times 10^{18} \mathrm{~m}^{-3}$ (blue solid), $2 \times 10^{19} \mathrm{~m}^{-3}$ (red solid), $2 \times 10^{20} \mathrm{~m}^{-3}$ (green solid), $2 \times 10^{21} \mathrm{~m}^{-3}$ (cyan solid) are used to calculate $\tau_{\mathrm{HCE}}^{\text {inc }}$ for each $n_{e}$. The relevant electron parameters $\left(n_{e} \approx 1.4 \times 10^{20} \mathrm{~m}^{-3}\right.$, $T_{e} \approx 5.5 \mathrm{eV}, n_{1} \approx 2.0 \times 10^{19} \mathrm{~m}^{-3}$ ) at cell 111 are donoted by the vertical dashed line in (c).

which is the main explanation for Eq. (40).

Additionally, it can be inferred, by comparing Figs. $8 \mathrm{~b}$ and $8 \mathrm{~d}$, that the distributions of $\Delta f_{e}^{\mathrm{rad}}$ both in Approaches $\mathrm{A}$ and $\mathrm{B}$ are quite similar when $T_{e}=\sim 5 \mathrm{eV}$. For low upstream collisionality cases $n_{u}<1.0 \times 10^{19} \mathrm{~m}^{-3}$, the electron temperature profile is quite flat from the stagnation point to the target, and the electron temperature in the near target region where radiation concentrates is high so that radiation sinks come mostly from thermal electrons. For medium and high collisionalities, although the target electron temperature can be very low compared to the ionization potential $I_{\mathrm{th}}$, e.g. $\sim 1 \mathrm{eV}$ for the case with $n_{u}=2.5 \times 10^{19} \mathrm{~m}^{-3}$, the radiation concentrates in the region with $T_{e}=4 \sim 6 \mathrm{eV}$. Therefore, the difference between the two approaches is not observable at any collisionality.

The plasma profiles achieved above in the scanned cases with pure deuterium correspond to ASDEX-Upgrade L-mode SOL plasmas where the upstream electron temperature $T_{u}=40 \sim 50 \mathrm{eV}$ and the target electron temperature varies with upstream collisionalities. To achieve H-mode plasma profiles, power input is increased to 
increase the stagnation point electron temperature $T_{u}$ to $\sim 80 \mathrm{eV}$, and carbon impurities are introduced as radiators to achieve the electron temperature variation by more than factor $10\left(T_{u} / T_{t} \approx 15\right)$. The two numerical schemes of removing electron cooling radiation are also applied for deuterium neutrals under the H-mode condition, while the atomic data for carbon are taken from the ADAS database for simplicity. As expectation, no observable differences appear in the simulation results.

The simulation results achieved above confirm the conclusion in [24] by series of self-consistent cases with a physical inelastic collision operator. We would like to remind again that the steady state profiles are rather insensitive to kinetic details of where (in which location of $f_{e}$ in velocity space) radiation sink is introduced since it is rather symmetric against $v_{\|}$. In future coupling runs, the uniform scheme for energy sources is suggested.

\section{Summary}

There are two kinds of effects that can be studied with the implementation of multiple inelastic collisions in KIPP: (1) effects of non-Maxwellian tails on effective deuterium ionization rates; (2) kinetic effects of electron cooling due to inelastic collisions on plasma profiles. The first was elucidated in [15], which showed that nonMaxwellian tails had little influence on rates. This work is mainly focused on the second effect, which requires the inelastic collision operator in the kinetic code KIPP. Various collisionalities have been investigated and it can be concluded that atomic physics effects on kinetic electron parallel transport are negligible compared to the overall effect of including effective kinetic factors instead of simple Braginskii-type fluid transport. This conclusion holds true for plasma conditions that vary from low collisionalities to high in which the profile in the density scan case with medium collsionalities correspond to ASDEX-Upgrade L-mode plasmas and the case with the $\mathrm{C}$ impurities correspond to ASDEX-Upgrade H-mode plasmas. However, full detachment, where the inelastic collision operator may play an important role, is not studied in this work since the coupling scheme has not worked well yet for detachment which showed oscillations and resulted in crashes [13]. The inelastic collision operator will be directly extrapolated to detachment cases once the oscillations are removed in the future.

Acknowledgements This work has been carried out within the framework of the EUROfusion Consortium and has received funding from the Euratom research and training programme 2014- 2018 under grant agreement No 633053. The views and opinions expressed herein do not necessarily reflect those of the European Commission.

\section{References}

[1] S. I. Braginskii, in Reviews of Plasma Physics (Consultants Bureau, New York, 1965), Vol. 1, Chap. Transport Processes In A Plasma, p. 205.

[2] R. Schneider et al., Contrib. Plasma Phys. 46, 3 (2006).

[3] G. J. Radford et al., Contributions to Plasma Physics 36, 187 (1996).

[4] M. E. Rensink et al., IAEA Technical Committee Meeting on Advances in Computer Modeling of Fusion Plasmas, Los Angeles (UCRL-JC-125076, United States, 1996), No. 28058080, p. 21.

[5] R. Chodura, Contrib. Plasma Phys. 32, 219 (1992).

[6] S. I. Krasheninnikov, Sov. Phys. JETP 67, 2483 (1988).

[7] S. I. Krasheninnikov, Physics of Plasmas 5, 74 (1993).

[8] A. V. Chankin, D. P. Coster, and G. Meisl, Contrib. Plasma Phys. 52, 500 (2012).

[9] A. V. Chankin and D. P. Coster, Contrib. Plasma Phys. 54, 493 (2014).

[10] A. V. Chankin and D. P. Coster, Journal of Nuclear Materials 463, 498 (2015).

[11] G. Meisl, A. V. Chankin, and D. P. Coster, Journal of Nuclear Materials 438, (2013).

[12] B. A. Trubnikov, in Reviews of Plasma Physics (Consultants Bureau, New York, 1965), Vol. 1, Chap. Particle Interactions in A Fully Ionized Plasma, p. 105.

[13] M. Zhao, A. V. Chankin, and D. P. Coster, Computational Physics Communications 235, 133 (2019).

[14] M. Zhao, Ph.D. thesis, Technische Universität München, München, 2018, http://pubman.mpdl.mpg.de/ pubman/item/escidoc:2590992/component/escidoc:2590995/IPP\%202018-10.pdf.

[15] M. Zhao, A. V. Chankin, and D. P. Coster, Plasma Physics and Controlled Fusion 61, 025019 (2019).

[16] R. Chodura, Contrib. Plasma Phys. 28, 303 (1988).

[17] R. Chodura, Contrib. Plasma Phys. 28, 325 (1988).

[18] R. Chodura, Contrib. Plasma Phys. 30, 153 (1990).

[19] D. P. Coster, Journal of Nuclear Materials 390, 826 (2009). 
[20] D. P. Coster, Contrib. Plasma Phys. 56, 790 (2016).

[21] D. Tskhakaya et al., Contrib. Plasma Phys. 48, 89 (2008).

[22] D. Tskhakaya et al., Journal of Nuclear Material 390, 335 (2009).

[23] S. E. Parker, R. J. Procassini, C. K. Birdsall, and B. I. Cohen, Journal of Computational Physics 104, 41 (1993).

[24] M. Zhao, A. V. Chankin, and D. P. Coster, Nuclear Materials and Energy 12, 819 (2017).

[25] H. P. Summers et al., Plasma Phys. Control. Fusion 48, 263 (2006).

[26] M. Capitelli et al., in Fundamental Aspects of Plasma Chemical Physics (Springer New York, New York, 2016), Vol. 85, Chap. Collisional-Radiative Models for Atomic Hydrogen Plasmas, p. 143. 